\title{
The Central Track Trigger of the DØ Experiment
}

Yuan $\mathrm{Hu}$, John Anderson, Robert Angstadt, Levan Babukhadia, Mrinmoy Bhattacharjee, Gerald Blazey, Fred Borcherding, Brian Connolly, Michael Cooke, Satish Desai, David Evans, Paul Grannis, Stefan Grünendahl, Carsten Hensel, Vivek Jain, Marvin Johnson, Miroslav Kopal, Stephan Linn, Juan Lizarazo, Yurii Maravin, Manuel Martin, Yildirim Mutaf, Carsten Nöding, Jamieson Olsen, Ricardo Ramirez-Gomez, Stefano Rapisarda, Kyle Stevenson, Makoto Tomoto, Brigitte Vachon, Thei Wijnen, Neal Wilcer, Graham Wilson, Qichun Xu, and Kin Yip

\begin{abstract}
The general purpose DØ collider detector, located at Fermi National Accelerator Laboratory, is operated in the high luminosity $\left(L=2 \times 10^{32} \mathrm{~cm}^{-2} \cdot \mathrm{s}^{-1}\right)$ and high-collision-rate environment (396 $\mathrm{ns}$ between beam crossings) of the upgraded Tevatron proton anti-proton accelerator. $D \emptyset$ uses a three-tiered trigger system to select events for offline storage and analysis. This paper describes the architecture and performance of the $D \emptyset$ central track trigger (CTT) system based on the new central fiber tracker, central preshower and forward preshower detectors, with emphasis on the interface to and integration with the second tier L2 Trigger system.
\end{abstract}

Index Terms-Fiber tracker, particle detector, Trigger.

\section{INTRODUCTION}

$\mathbf{T}$ HE D $\varnothing$ detector is a spectrometer at the Tevatron Collider at Fermi National Accelerator Laboratory [1], [2]. Proton and anti-proton bunches are accelerated to energies of about 1 $\mathrm{TeV}$, circulating in opposite directions and colliding in the center

Manuscript received November 14, 2003; revised May 4, 2004, and July 7, 2004.

Y. Hu, L. Babukhadia, M. Bhattacharjee, S. Desai, P. Grannis, and Y. Mutaf are with the Department of Physics and Astronomy, State University of New York, Stony Brook, NY 11794 USA (e-mail: huy@fnal.gov).

J. Anderson, R. Angstadt, F. Borcherding, S. Grünendahl, M. Johnson, Y. Maravin, J. Olsen, S. Rapisarda, M. Tomoto, B. Vachon, and N. Wilcer are with the Fermi National Accelerator Laboratory, Batavia, IL 60510-0500 USA.

G. Blazey and M. Martin are with the NICADD/NIU, Northern Illinois University, DeKalb, IL 60115 USA.

B. Connolly and S. Linn are with the Physics Department, Florida State University, Tallahassee, FL 32306 USA.

M. Cooke is with the TW Bonner Nuclear Lab, Rice University, Houston, TX 77251-1892 USA.

D. Evans is with the Department of Physics, Lancaster University, Lancaster, Lancashier LA1 4YB, U.K.

C. Hensel and G. Wilson are with the Department of Physics and Astronomy, University of Kansas, Lawrence, KS 66045 USA.

V. Jain is with the Brookhaven National Laboratory, Upton, NY 11973-5000 USA.

M. Kopal is with the Department of Physics and Astronomy, University of Oklahoma, Norman, OK 73019 USA.

J. Lizarazo and R. Ramirez-Gomez are with the Departamento de Fisica, Universidad de Los Andes, Apartado Aereo 4976, Bogota, Colombia.

C. Nöding is with the Albert-Ludwigs-Universität Freiburg, Physikalisches Institut, 79104 Freiburg, Germany.

K. Stevenson is with the Department of Physics, Indiana University, Bloomington, IN 47405 USA.

T. Wijnen is with the University of Nijmegen, 6546 ED Nijmegen, The Netherlands.

Q. Xu is with the Department of Physics, University of Michigan, Ann Arbor, MI 48109 USA.

K. Yip is with Brookhaven National Laboratory, Upton, NY 11973-5000 USA.

Digital Object Identifier 10.1109/TNS.2004.836090

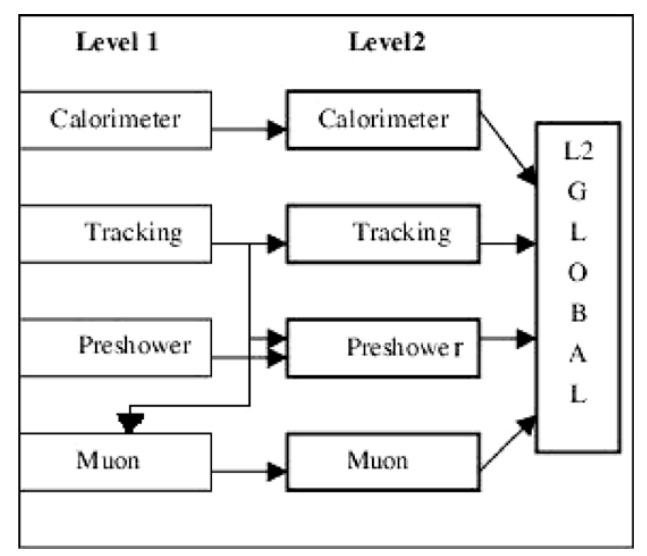

Fig. 1. Block diagram of $\mathrm{L} 1$ and $\mathrm{L} 2$ trigger components.

of the DØ detector. The collisions happen every $396 \mathrm{~ns}$, far exceeding the rate of writing events on tape for offline analysis. This extremely high rate requires the $\mathrm{D} \emptyset$ detector to be operated with a significantly enhanced trigger system to quickly analyze detector data and select only the most interesting physics events to be saved for offline analysis.

The trigger for $\mathrm{D} \emptyset$ is divided into three successive levels, each incorporating more rigorous filter algorithms [3], [4]. A hardware/firmware-based dead-timeless Level 1 (L1) trigger searches for patterns consistent with electrons, muons, taus and jets to reduce the raw collision rate to $10 \mathrm{kHz}$. Level 2 (L2) uses, firstly, individual subdetector specific preprocessors to combine L1 objects and additional detector information and then, a global stage to test for correlations between subdetectors, and further reduces the event rate to $1 \mathrm{kHz}$. Level 3 (L3) is a computer farm that uses software algorithms for particle identification after simple event reconstruction. The final rate of writing events to tape is about $50 \mathrm{~Hz}$. Fig. 1 shows the block diagram of L1 and L2 trigger components.

The central track trigger (CTT) system based on the DØ central track subdetectors is one of the major components of the $D \varnothing$ trigger [5], [6]. The CTT reconstructs trajectories of charged particles and preshower clusters using fast discriminator data provided by scintillator-based central track subdetectors. At L1, the CTT uses hardware/firmware to process data and make L1 trigger decisions, trigger elements known as trigger terms. The L2 CTT includes three preprocessors and provides more detailed information which is later used by the $\mathrm{L} 2$ global processor to make trigger decisions based on the correlations between the central track system and other subdetectors. 


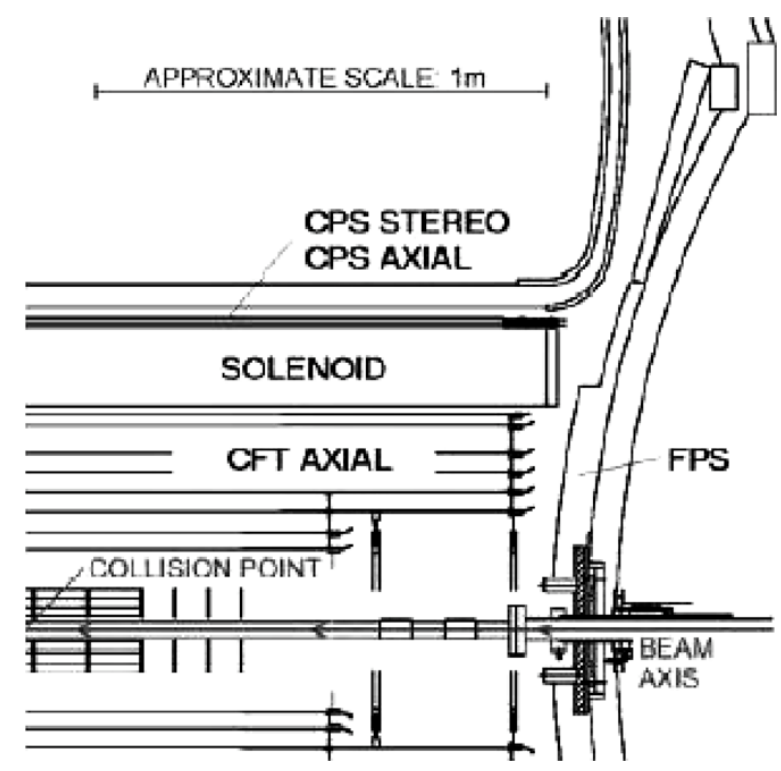

Fig. 2. The axial view of one quarter of the $D \emptyset$ central track system.

\section{The Central Track System of DØ}

The DØ central track system includes three detectors, shown in Fig. 2: the central fiber tracker (CFT) [7], the central preshower (CPS) [8], and the forward preshower (FPS) [9].

The CFT is composed of $77 \mathrm{k}$ scintillating fibers mounted on eight carbon-composite cylinders concentric with the beam line. Half of these fibers are mounted axially (parallel to the beam line), and the remainder are mounted with a small stereo angle. The innermost cylinder is $20 \mathrm{~cm}$ from the beam and the outermost $55 \mathrm{~cm}$. A 2 Tesla super conducting solenoid magnet is adjacent to the outer CFT cylinder. The CFT provides coverage down to about $22^{\circ}$ from the beam axis, corresponding to the pseudo-rapidity $(\eta)$ region $|\eta|<1.7$.

The CPS is mounted just outside the solenoid (radius $70 \mathrm{~cm}$ ), and has three cylindrical layers with a total of $7 \mathrm{k}$ triangular scintillator strips with embedded wavelength shifting fibers. One of these layers is axial and the other two are stereo layers. The CPS provides additional means of identifying electrons and photons with coverage down to about $31^{\circ}$ from the beam axis $(|\eta|<$ 1.3).

The FPS detector is constructed of $15 \mathrm{k}$ triangular scintillator strips similar to CPS, but oriented in the transverse plane. The FPS is divided into two halves and each half is positioned near either the south or north end calorimeters with coverage from $25^{\circ}$ down to $9^{\circ}$ from the beam axis $(1.5<|\eta|<2.5)$. The FPS is comprised of four stereo layers with a lead radiator in the middle. The upstream two layers are called MIP layers, while the downstream two are called shower layers. It provides additional electron and photon identification in the forward region.

When particles pass through the central track subdetectors, the plastic scintillators transform a small fraction of the deposited energy into visible light. About 100000 optical waveguides deliver this light to visible light photon counters (VLPCs) [10], [11]. The VLPCs are light-sensitive solid-state photo-multipliers that convert the light into electrical signals. The analog front-end (AFE) boards [12] located on the VLPC cryostat split the electrical signal into two parts, one part is discriminated and sent out to the trigger system, the other part is stored in an analog pipeline for later (after L1 accept) digitization and readout after an L3 accept [13].

\section{Central Track Trigger System}

The overall CTT system architecture is depicted in Fig. 3. The CTT system is comprised of three subsystems: CFT/CPS axial, CPS stereo, and FPS, which process signals in parallel. Of these, the CFT/CPS axial and FPS subsystems form L1 trigger terms which are sent to the $D \varnothing$ trigger framework. All three subsystems participate in L2/L3 readout by sending track and cluster lists to various preprocessor and readout crates.

The AFE boards send the discriminated signals to chains of digital front-end (DFE) boards over point-to-point low voltage differential signal (LVDS) links every $132 \mathrm{~ns}$. All of the DFE boards in the CTT system, such as the digital front-end board for CFT/CPS axial (DFEA), digital front-end board for CPS stereo (DFES), digital front-end board for FPS (DFEF), board processing CFT/CPS axial information for one detector octant (CTOC), board processing CFT/CPS axial information for one detector quadrant (CTQD), board reformatting CFT/CPS axial information in the sextant geometry of the silicon microstrip track subdetector (STOV), STSX (similar to STOV), board processing FPS information for one detector quadrant (FPSS), board forming the CFT/CPS axial L1 trigger And/Or terms (CTTT), and board forming the FPS L1 trigger And/Or terms (FPTT), are built using a common $6 \mathrm{U} \times 320 \mathrm{~mm}$ motherboard that supports two different layouts of daughterboard with different sizes and numbers of field programmable gate arrays (FPGAs). A more detailed description of the structure and function of the DFE boards can be found in [14] and references therein. The MIXER is used to map the AFE outputs (cylinder layer geometry) on the DFEA inputs (sector geometry).

For the L1 decision, counts of tracks (clusters) found in each DFEA (DFEF) boards are passed to CTOC (FPSS) boards. The CTOC (FPSS) boards sum these counts of tracks (clusters) and pass the information to the CTTT (FPTT), where the L1 trigger terms are produced and sent to the $D \emptyset$ trigger framework in each beam crossing. In addition, DFEA boards send track information to the $\mathrm{L} 1$ muon trigger.

Once an event is accepted at L1, each AFE board will jump back to this event in the analog buffers and digitize the fiber data with 8-bit resolution. When the L1_ACCEPT control bit embedded in the data stream reaches the DFEA, DFES, and DFEF boards, these then send a sorted list of found tracks and clusters to the next tiers along each chain. The lists are sorted by CTOC, CTQD, and FPSS boards and passed downstream to preprocessor crates for more detailed analysis. The STOV and STSX reformat the tracks from the DFEA in the sextant geometry of the silicon microstrip track (SMT) subdetector, and send them to the L2 silicon track trigger (STT) to find the impact parameter of each track.

\section{LEVEL 2 PREPROCESSORS}

During Level 2 processing, the DFE boards send track and cluster information to the preprocessors using optical fiber links (GLink). The preprocessors handle (in parallel) data specific to 


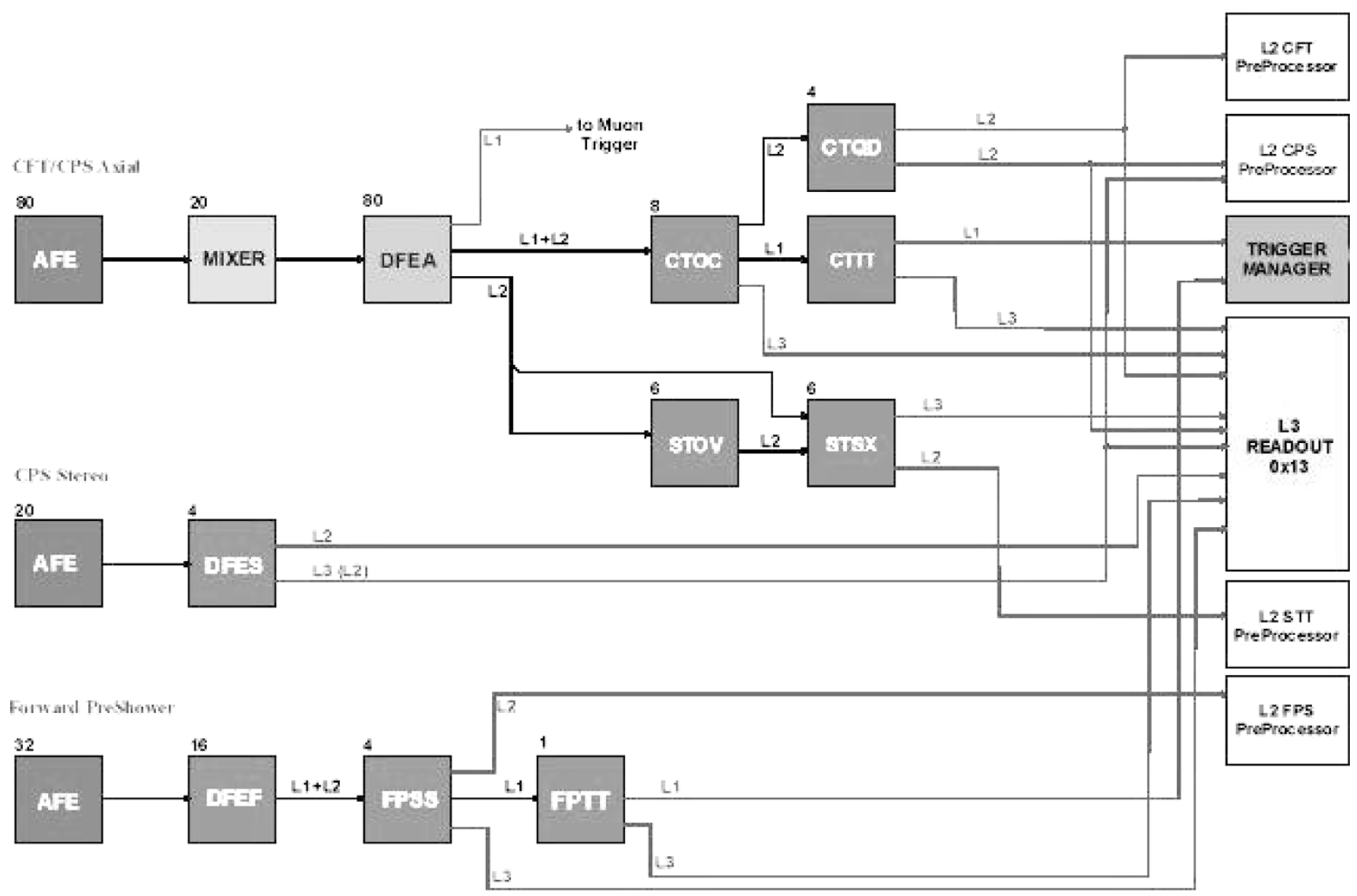

Fig. 3. The schematic diagram of CTT system.

particular detectors and pass lists of objects to the L2 Global processor (Fig. 3). The CTT includes three preprocessors: L2CFT, L2CPS, and L2FPS.

Each preprocessor occupies a 64-bit VME for Physics $9 \mathrm{U}$ crate with an auxiliary "Magic Bus" [15] backplane. The major devices in this crate, as well as the data flow, are illustrated in Fig. 4.

Signals from the DFE boards are brought to the fiber input converters (FICs) through the VRB transition module (VTM) card in the backplane. The FIC transforms the optical input signal into electrical signals and sends those signals to the Magic Bus transceivers (MBTs) via Cypress Hot Links.

The MBT card [16] is the main I/O building block for the crate. The main function of the MBT card is to receive inputs and broadcast the data to two processors, which are refered to as the "Administrator" processor and the "Worker" processor, through the Magic Bus. The MBT also has two Hotlinks transmitters, used by preprocessors to send results to the L2 Global. In addition, the MBT supports various control, testing, and monitoring functions.

The Administrator processor gets the data broadcast from the MBT, coordinates assignment of event buffers, manages readout to L2 Global, checks the data integrity and controls the output of monitoring data. The trigger algorithm programmed in $\mathrm{C}++$ runs on the Worker processor. These two processors reside in the Beta processor board [17]. This board is basically a Single

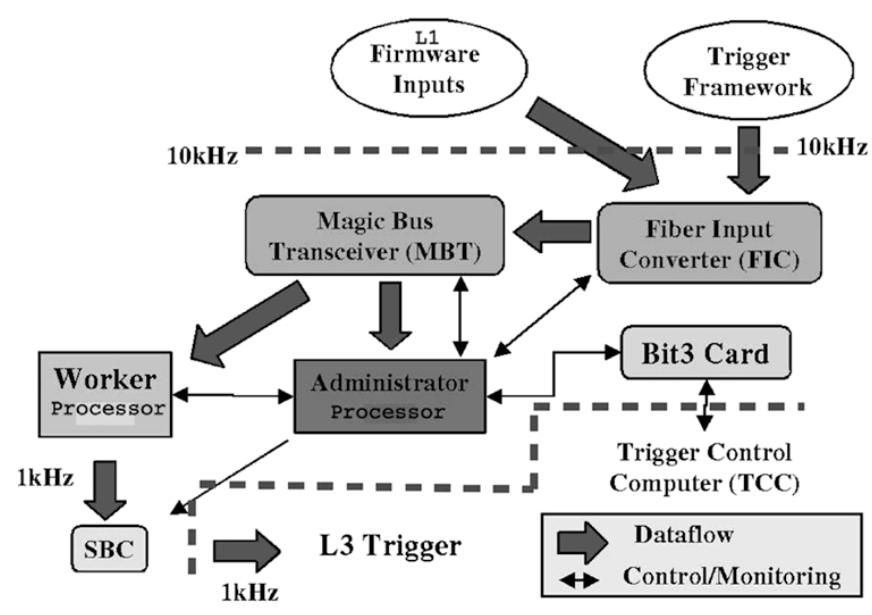

Fig. 4. The L2 preprocessor schematic plot.

Board Computer (with 64-bit PCI interface chip, PCI card and two 1 GHZ PIII CPUs on the board).

The Bit 3 Card is a dual-port-memory (DPM) used for the download of run-time parameters and the reporting of monitoring data to the trigger control computer (TCC). The single board computer (SBC) is used to readout the data in the worker over the VME bus to the level-3 trigger system.

The format of the data sent from the L2 preprocessors to L2 Global includes a header specifying the format, length of header 


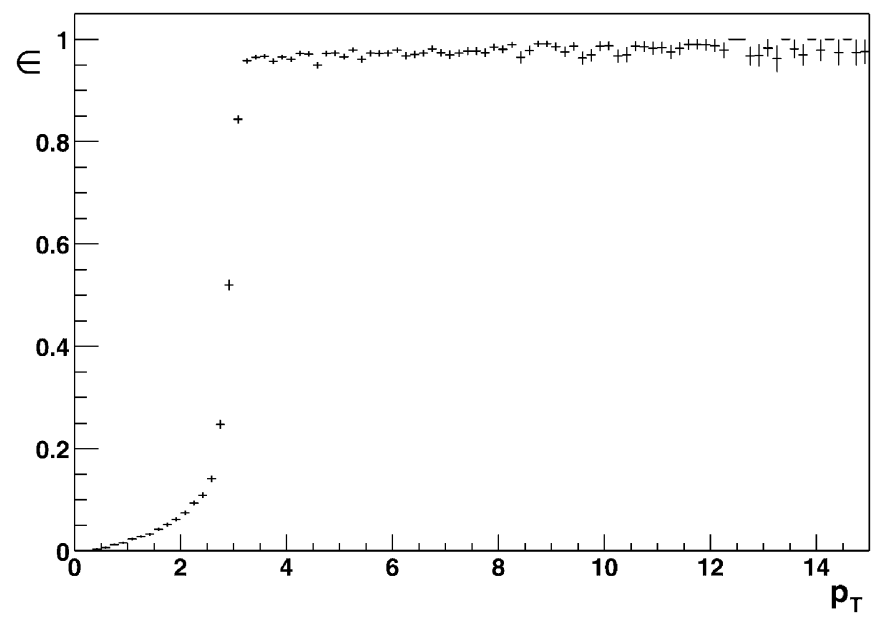

Fig. 5. The efficiency of L1 CTT trigger term $\operatorname{TTK}(2,3)$, the horizontal axis is the $p_{T}$ of the second most energetic track in units of $\mathrm{GeV} / \mathrm{c}$.

and objects, and an event number in the header and trailer, and also parity information in the trailer, allowing for verification of the transfer. Objects are fixed length for a given preprocessor, and an integral number of 32-bit (4-Byte) longwords. The data sources must pad the transfer after the trailer to form a number of longwords divisible by 4 , since the Magic Bus is 16 Byte wide.

\section{CTT PERFORMANCE}

Many of the physics topics of $\mathrm{D} \emptyset$, such as the searches for the Higgs boson, top quark property studies, and the measurements of electroweak processes, rely heavily on triggering on events with high $p_{T}$ tracks. Other physics processes, like studies of signatures for "physics beyond the Standard Model," and the measurements of B physics, can benefit from triggering on electrons in the forward region. The CTT is very crucial to triggering on these events. In order to qualify the CTT performance, we compare trigger information provided by the CTT to the offline reconstructed data.

\section{A. The CTT Level 1 Performance}

At Level 1, the function of the CFT/CPS Axial subsystem is to report the number of tracks within four predefined $p_{T}$ bins, the number of tracks associated with CPS clusters, and the number of isolated tracks. Fig. 5 plots the efficiency of the $\operatorname{TTK}(2,3)$ trigger term, which is set for events with two or more tracks above a $p_{T}$ threshold of $3 \mathrm{GeV} / \mathrm{c}$. This plot is obtained by using unbiased events with respect to the studied trigger term, and requiring all offline tracks to be constructed in good quality. It is apparent that the L1 CTT can trigger on this kind of event with an efficiency of almost $100 \%$ at the plateau.

The minimal difference of azimuthal angle between $\mathrm{L} 1$ tracks triggered by the CTT and the offline reconstructed electrons in a $Z \rightarrow e^{+} e^{-}$sample is shown in Fig. 6. The $\mathrm{L} 1$ track is matched with an offline electron if they both lie in the same trigger sector $\left(|\delta \phi|<2.25^{\circ}\right)$. About $92 \%$ matching efficiency indicates that the high $p_{T}$ electrons in the $Z \rightarrow e^{+} e^{-}$sample have been highly efficiently found by the L1 CTT system. This type of object matching is currently being studied for implementation at L2.

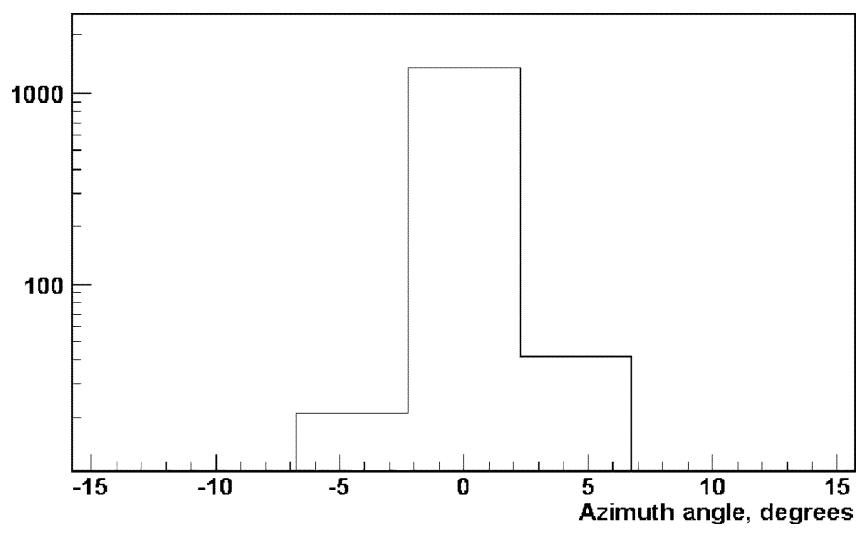

Fig. 6. The minimal difference of azimuthal angle between L1 tracks and offline reconstructed electrons in a $Z \rightarrow e^{+} e^{-}$sample.

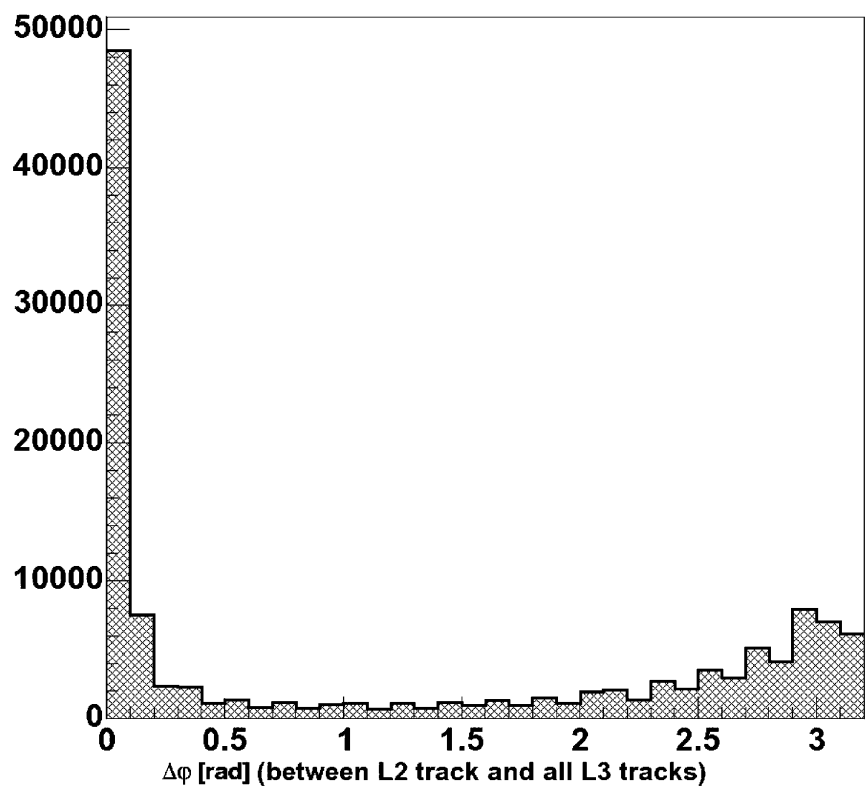

Fig. 7. The minimal difference of azimuthal angle between L2 tracks and L3 tracks in radians.

More discussion of the L1 CTT performance can be found in an accompanying paper presented at this conference [18].

\section{B. The CTT Level 2 Performance}

For Level 2, the CFT preprocessor is supposed to give a detailed list of tracks with information on isolation, sign of the charge, global azimuthal angle, and refined $p_{T}$ value. These tracks are sorted descending in $p_{T}$, and also labeled with the associated CPS clusters. The L2 CPS preprocessor and the L2 FPS preprocessor try to provide the list of preshower clusters which are above a threshold that can be modified depending on which electron energy range one wishes to trigger on. The global coordinates (the rapidity $\eta$ and the azimuthal angle $\phi$ ) and the width of those preshower clusters are also reported. Preliminary results from the newly commissioned L2 CFT are displayed in Figs. 7 and 8.

Fig. 7 shows the minimal difference of azimuthal angle between a track found at Level 2 with $p_{T}>3 \mathrm{GeV} / \mathrm{c}$ and tracks found at Level 3 with $p_{T}>3 \mathrm{GeV} / \mathrm{c}$. Level 3 uses roughly the same algorithm as used in offline reconstruction to find tracks. 


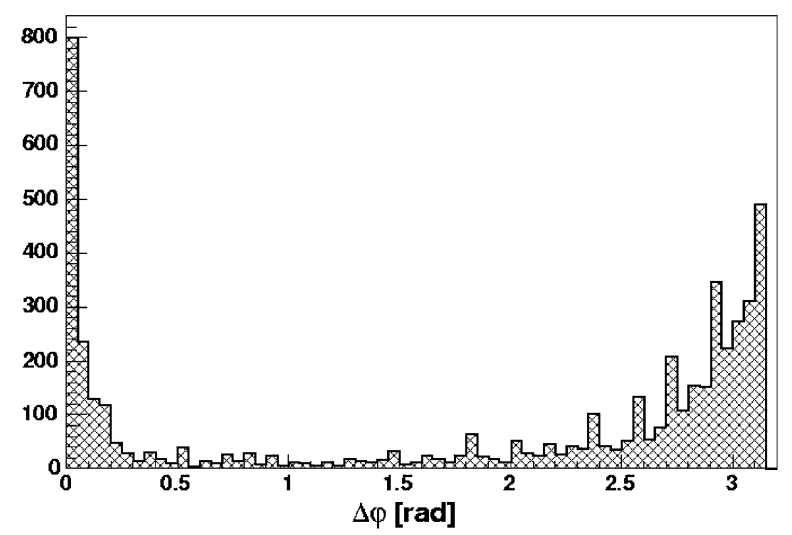

Fig. 8. The difference of azimuthal angle between L2 tracks and L2 electromagnetic objects in radians.

The peak at the small value region shows that most of the high $p_{T}$ tracks reported by the L2 CFT preprocessor can be matched with offline tracks and L2 CFT has very good performance in finding high $p_{T}$ tracks in events.

The azimuthal angle difference between L2 tracks and L2 electromagnetic objects which were found by the Level 2 calorimeter preprocessor is plotted in Fig. 8. The peak at zero indicates that the found tracks and electromagnetic objects are highly correlated (the peak near $\phi=\pi$ is due to other tracks in the event that balance the transverse momentum), and shows that L2 global can trigger on electrons highly efficiently based on this correlation.

\section{SUMMARY}

The CTT system is a very important component of the DØ trigger. The L1 CFT/CPS axial subsystem has been operating steadily, and its trigger information is being utilized for physics triggers. Offline studies demonstrate that this subsystem is efficient in finding and triggering on charged particle tracks. The L2 CFT and CPS subsystems are online since August 2003. Preliminary results indicate these two subsystems are of good per- formance in finding tracks and clusters which can be used by the L2 Global processor to make trigger decisions in correlation with other subdetectors.

All hardware for the FPS subsystem is in place and trigger algorithms for both firmware and software are currently being developed.

\section{REFERENCES}

[1] S. Abachi et al., "The DØ detector," Nucl. Instrum. Methods, vol. A338, pp. 185-253, 1994.

[2] "The DØ Upgrade: The Detector and Its Physics," DØ Collaboration, Fermilab Pub-96/357-E, 1996.

[3] G. C. Blazey. The DØ RunII Trigger. [Online]. Available: http:niuhep.physics.niu.edu/ blazey/upgrade.html

[4] F. Borcherding, S. Grünendahl, M. Johnson, J. Olsen, and K. Yip, "A first level tracking trigger for the upgraded DØ detector," IEEE Trans. Nucl. Sci., vol. 46, pp. 359-364, June 1999.

[5] The DØ SciFi Tracking Trigger Technical Design Report, DØ Note 3551. [Online]. Available: http://www-d0.fnal.gov/d0notes_forms/notes.html

[6] L. Babukhadia and M. Martin. Track and Preshower Digital Trigger in DØ. [Online]DØ Note 3980

[7] "The DØ Upgrade: Central Fiber Tracker, Technical Design Report," DØ CFT working group, DØ Note 4164.

[8] M. Adams et al., "Design Report of the Central Preshower Detector for the DØ Upgrade,", DØ Note 3014.

[9] A. Gordeev et al., "Technical Design Report of the Forward Preshower Detector for the DØ Upgrade,", DØ Note 3445.

[10] M. D. Petroff, M. G. Stapelbroek, and W. A. Kleinhans, Appl. Phys. Lett., vol. 51, pp. 406-408, 1987.

[11] D. Adams et al., "First large sample study of visible light photon counters (VLPC's)," Nucl.Phys. B (Proc. Suppl.), vol. 44, pp. 340-348, 1995.

[12] J. Anderson. DØ Central Fiber Tracker 8-MCM Analog Front-End Board, Design Specification. [Online]. Available: http://d0server1. fnal.gov/users/janderson/Public_Eng_Notes/default.html

[13] T. Zimmerman, M. Sarraj, and R. J. Yarema, "Design of an advanced readout chip for silicon strip detectors," IEEE Trans. Nucl. Sci., vol. 40, pp. 736-739, Aug. 1993.

[14] J. Olsen et al., "The DØ central track trigger," IEEE Trans. on Nucl. Sci., to be published.

[15] D. Edmunds, S. Gross, P. Laurens, J. Linnemann, and R. Moore, "Technical Design Report for the Level 2 Global Processor,”, DØ Note 3402.

[16] The Magic Bus Transceiver Specification. [Online]. Available: http:// www.pa.msu.edu/hep/d0/12/data_transfer/mbt_description.txt

[17] The Beta Processor Board Specification. [Online]. Available: http:// galileo.phys.virginia.edu/ rjh2j/l2beta

[18] Y. Maravin, "The DØ central tracking trigger," in Proc. IEEE/NNS-MIC, 2003. 